in vivo $35: 3591-3596(2021)$

doi:10.21873/invivo.12664

\title{
Malignant Struma Ovarii Treated With Cytoreductive Surgery (CRS) and Hyperthermic Intraperitoneal Chemotherapy (HIPEC)
}

\author{
MIKLOS ACS ${ }^{1}$, SEBASTIAN HÄUSLER ${ }^{2}$, HAMID-REZA LIGHVANI $^{3}$, JOZEF ZUSTIN ${ }^{4,5}$ and POMPILIU PISO ${ }^{1}$ \\ ${ }^{1}$ Department of General and Visceral Surgery, Krankenhaus Barmherzige Brüder, Regensburg, Germany; \\ ${ }^{2}$ Department of Obstetrics and Gynecology, \\ Krankenhaus Barmherzige Brüder, Klinik St. Hedwig, Regensburg, Germany; \\ ${ }^{3}$ Department of Nuclear Medicine, Krankenhaus Barmherzige Brüder, Regensburg, Germany \\ ${ }^{4}$ Gerhard Domagk Institute of Pathology, University Medical Center Muenster, Muenster, Germany; \\ ${ }^{5}$ Institute of Pathology Regensburg, Regensburg, Germany
}

\begin{abstract}
Background/Aim: Malignant struma ovarii is an extremely rare tumor entity among ovarian tumors. In the presence of ascites and peritoneal metastases, the preoperative appearance may resemble the most common epithelial ovarian carcinoma (EOC) and accordingly, the surgical therapy may be identical if a preoperative histology diagnosis is not possible. The objective of this case report is to present a patient with histopathologically confirmed malignant struma ovarii who underwent cytoreductive surgery and hyperthermic intraperitoneal chemotherapy (CRS and HIPEC) with the aim of complete tumor resection. Case Report: This study reports on a patient with preoperatively proven peritoneal metastasis of an $18 \mathrm{~cm}$ ovarian tumor with large struma ovarii and papillary thyroid carcinoma within the struma, who was treated with CRS and HIPEC after neoadjuvant chemotherapy. Conclusion: This disease has a significantly better prognosis than EOC, however, HIPEC could provide an additional effect in examining the presence of peritoneal metastasis.
\end{abstract}

Struma ovarii is a monodermal teratoma among the germ cell tumors of the ovary mainly consisting of thyroid tissue

This article is freely accessible online.

Correspondence to: Miklos Acs, Department of General and Visceral Surgery, Krankenhaus Barmherzige Brüder, Prüfeninger Strasse 86, 93049 Regensburg, Germany. Tel: +49 9413692201 , Fax: +499413692206, e-mail: miklos.acs@barmherzigeregensburg.de

Key Words: Malignant struma ovarii, peritoneal carcinosis, HIPEC, ovarian cancer.
(>50\% of overall tissue) (1). This disease was first described by Gottschalk in 1899 (2). Struma ovarii is a very rare tumor, which represents $1 \%$ of all ovarian tumors and $2-5 \%$ of all ovarian teratomas $(3,4)$.

Out of these, $5 \%$ can transform into malignant tumors (5). The clinical presentation is usually non-specific and difficult to recognize and is mostly discovered incidentally according to pathological findings. Due to its rarity, the perioperative management is not uniform and not completely clarified to date. There is no case report in the literature in which HIPEC has been performed on malignant struma ovarii. Herein a patient with malignant struma ovary of papillary carcinoma histology is presented. She was treated with cytoreductive surgery (CRS) and hyperthermic intraperitoneal chemotherapy (HIPEC) after preoperatively proven peritoneal metastases and ascites in our tertiary referral center.

\section{Case Report}

A 61-year-old female patient was admitted, in January 2013, to an external hospital by her general practitioner with ascites that had been present for 2 months. Massive ascites was found, of which 6.3 liters were punctured. Despite immunohistochemical work-up (neither calretinin-positive mesothelium nor HEA-positive atypical cells could be detected), the cytological examination revealed no evidence of malignancy in the very cell-poor cell pellet. With a suspected malignant process and significant elevated CA-125 tumor marker of 1,041.7 IU/ml (reference range: 0-35 IU/ml), the patient was admitted to our clinic. A CT scan of the thorax and abdomen revealed an urgent suspicion of ovarian carcinoma with a $12 \times 5 \times 9.5 \mathrm{~cm}$ inhomogeneous, cystic, solid, partly calcified mass originating from the left ovary, massive ascites, and partly nodular, partly plate-like thickening of the 
omentum majus with an urgent suspicion of peritoneal carcinomatosis (Figure 1). In addition, there were a total of 4 cystic liver lesions in segments II, VI and VIII with a size up to $1.8 \mathrm{~cm}$. At the interdisciplinary tumor conference, the decision was initially made in favor of chemotherapy with 3 cycles of carboplatin/paclitaxel with intermediate staging during the course of treatment assuming the presence of liver metastases and due to the peritoneal carcinomatosis, which was considered initialy inoperable. The patient was scheduled for an intravenous chemotherapy with the carboplatin (AUC 5) and paclitaxel $\left(175 \mathrm{mg} / \mathrm{m}^{2}\right)$ regimen. An intermediate CT staging after 3 cycles of chemotherapy in March 2013 showed a significant decrease in the amount of ascites. The pelvic tumor manifestations, partly inhomogeneously solid, partly solid, partly cystic were identical in the course with still existing peritoneal carcinomatosis without organ metastases (Figure 2). The atypical cystic masses of the liver were assessed as non-malignant during the course of the disease. The patient received a further 3 cycles out of a total of 6 cycles of chemotherapy. After completion of the neoadjuvant chemotherapy in May 2013, another CT was performed, which showed a largely constant tumor mass in the pelvis with no new intraabdominal aspects. The laboratory value of CA125 also showed a good response of $323 \mathrm{IU} / \mathrm{ml}$ in March 2013 and $198 \mathrm{IU} / \mathrm{ml}$ in May 2013. At another tumor conference in June 2013, the indication for laparotomy was given with an attempt to achieve complete tumor resection with cytoreductive therapy, and subsequent hyperthermic intraperitoneal chemotherapy to target any residual microscopic disease. During the operation and HIPEC preparation, the patient gave her verbal and written consent for future processing and anonymized use of her medical data, also for scientific purposes. The operation was performed in June 2013. Intraoperatively, an $18 \mathrm{~cm}$ polycystic tumor was found starting from the left ovary as well as tumor-suspicious retraction in the area of the mesorectum as solitary peritoneal carcinomatosis. There was no evidence of peritoneal carcinomatosis in the upper abdomen, no tumor evidence specifically subphrenically on either side, neither in the liver, except for the already known macroscopically inconspicuous cysts. Likewise, the omentum majus appeared without macroscopic evidence of tumor. Tumor resection with hysterectomy and adnexectomy took place on both sides as well as resection of the retraction in the mesorectum and Douglas pouch while sparing the rectum. Omentectomy and pelvic and paraaortic lymphadenectomy were performed, as it was suggested by the respective national guidelines at the time of the surgery. Besides these procedures, partial parietal peritonectomy on both sides in the mid- and lower abdomen, followed by bidirectional hyperthermic intraperitoneal chemotherapy with cisplatin $75 \mathrm{mg} / \mathrm{m}^{2}(135.75 \mathrm{mg})$ and doxorubicin $15 \mathrm{mg} / \mathrm{m}^{2}(27.15 \mathrm{mg})$ for 60 minutes were performed as well. After the operation, a macroscopically complete tumor resection was conducted. The patient was treated postoperatively in our surgical intensive care unit for three days, and the course passed without any complications. The further course took place at the normal ward similarly without any complications. The histological result revealed an $18 \mathrm{~cm}$ ovarian tumor of the left adnexa consisting of struma ovarii and a $1 \mathrm{~cm}$ papillary thyroid carcinoma within the struma (Figure 3), which was resected in sano. No other teratoma-like differentiation was detectable. The immunohistochemical thyroglobulin was found positive, thyroid transcription factor-1 (TTF1) was positive, WT1 negative, p53 negative, Ki67 with only low proliferation activity. The removed peritoneal tissue and omentum preparations showed only significant fibrosis and scarring with no evidence of peritoneal carcinomatosis. The pelvic and paraaortic lymph nodes were also all free of tumor infiltration. The final histology revealed a FIGO stage IA disease (TNM: T1a, N0, M0). A BRAF mutation was not present. Postoperatively, the patient medical history was presented again at the interdisciplinary tumor conference, where thyroid diagnostics and completion of staging by whole-body scintigraphy within 3 months were recommended. Additive thyroid therapy (thyroidectomy and 131I therapy) would have only been recommended in case of $>\mathrm{T} 3$ tumor, BRAF mutation or metastasis. The following thyroid gland clarification took place in September 2013. The thyroid sonography revealed a circumscribed echogenic nodule formation of $1 \times 1 \times 1.5 \mathrm{~cm}$ with coarse scaly calcification basally in the left lobe in the sense of regressive struma uninodosa. Furthermore, the thyroid scintigraphy revealed an inhomogeneous, diffuse, clearly reduced accumulation of the thyroid gland without evidence of overactive zones, and without hypofunctional nodes (Figure 4). The uptake was $0.5 \%$. Thyroid serology showed a euthyroid state. Overall, thyroid diagnostics showed no evidence of thyroid neoplasia. Furthermore, the patient was advised to undergo regular follow-up with serum thyroglobulin determination and thyroid sonography, which she did not attend for unknown reasons. Since the surgery and the subsequent chain of diagnostics, there has been no contact with a physician, only a telephone follow-up by the general practitioner, which revealed no evidence of any symptoms after 8 years.

\section{Discussion}

Struma ovarii is difficult to diagnose on clinical basis and with imaging techniques, mostly discovered incidentally after pathological reprocessing. As in our case, malignant struma ovarii may appear with a picture of clinical mimicry, resembling the most common EOC in advanced stages on imaging with peritoneal carcinomatosis. But even with disease confined to the ovary on preoperative CT with elevated CA-125 levels, it is not possible to differentiate 


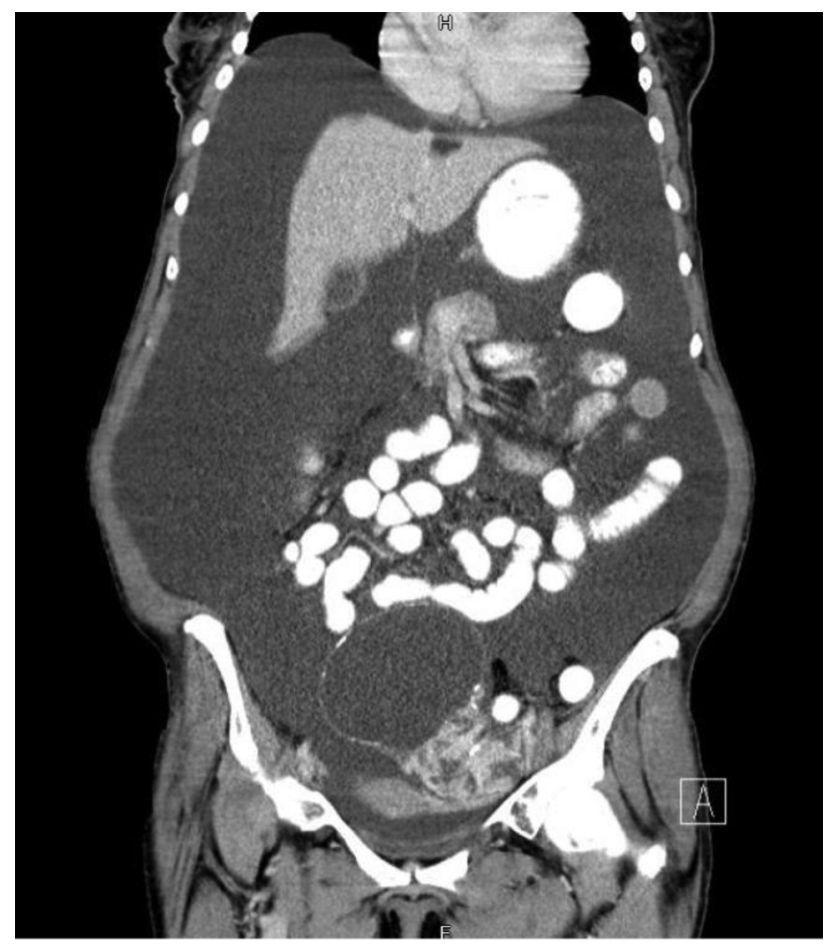

Figure 1. Initial finding: large tumor in the pelvis with pronounced ascites. Computed tomography (CT) image.

between the much more common histological subtypes as preoperative punctures are outdated methods. Due to the very rare occurrence of hyperthyroidism and the fact that clinically or biochemically manifest hyperthyroidism occurs in less than $5-8 \%$ of cases (6), preoperative hormone diagnostics reveal no pathological findings in general. As a result, if no frozen section is performed intraoperatively, the therapy can be identical to epithelial ovarian cancer including HIPEC.

Furthermore, a recent retrospective study of 125 cases found ascites in only $17.6 \%$ and elevated levels of CA-125 in $36.4 \%$ of the cases (7). Another study of 88 patients found that ascites occurred without evidence of tumor in every patient where cytological reports were available, making preoperative diagnosis even more difficult (8).

At present, no standard guidelines exist for surgical management for malignant struma ovarii $(4,7)$. In a review by $\mathrm{Li}$ et al. unilateral adnexectomy was performed in $33.6 \%$ of the cases, $21.6 \%$ of patients underwent abdominal hysterectomy with bilateral adnexectomy, and only $17.6 \%$ underwent debulking surgery. Bilateral adnexectomy (5.6\%) and ovarian cystectomy $(8 \%)$ were performed in the minority of the cases. Examining the recurrence-free survival (5 year and 10 year RFS $27.1 \%$ and $35.2 \%$, respectively) and overall

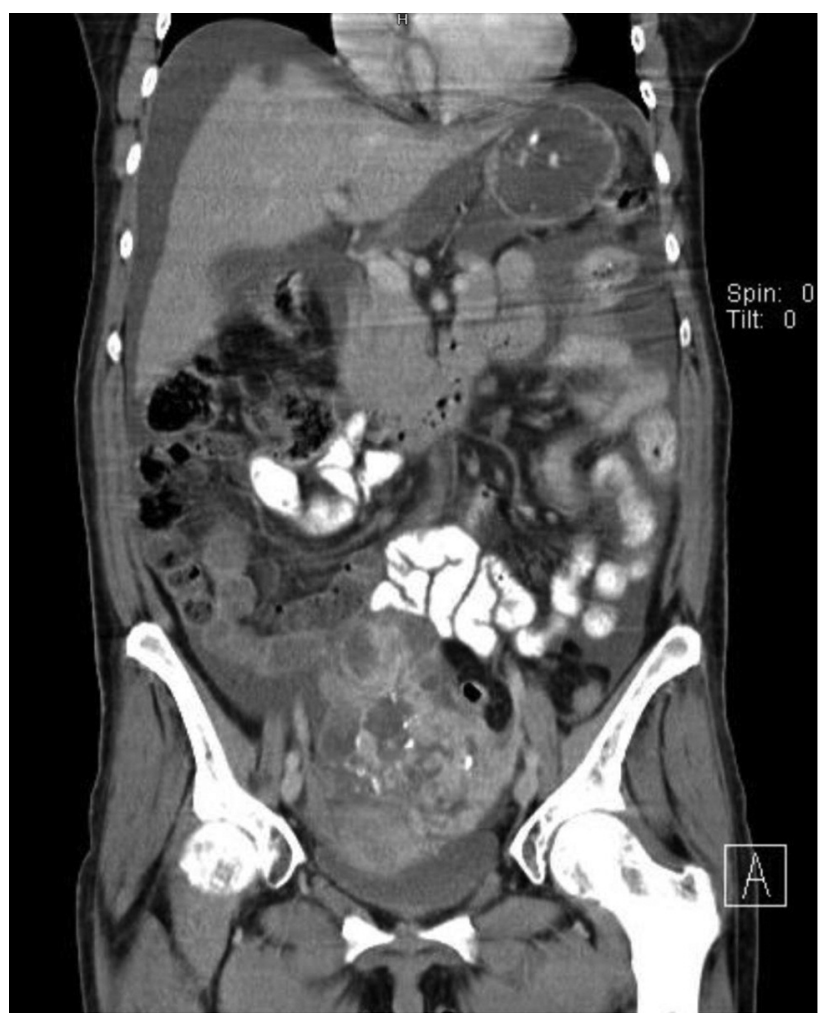

Figure 2. Significant decrease of ascites with the same tumor manifestation in the pelvis after 6 cycles of systemic chemotherapy. Computed tomography (CT) image.

survival, excellent survival rate was found regardless of the surgical management applied (5 year, 10 year, and 20 year OS rates were $95.3 \%, 88.7 \%$ and $88.7 \%$, respectively) (7). As other authors have also suggested, in our treatment decision there was more extensive surgical intervention in case of postmenopausal patients than when fertility prevention is desired at younger patients at a reproductive age $(6,9)$. Our approach of administering primary systemic chemotherapy within initially not resectable ovarian cancer and performing interval tumor debulking with additional HIPEC was recently confirmed in a study by van Driel (10). Furthermore, application of hyperthermic intraperitoneal chemotherapy was considered in our patient for the reason of suspected peritoneal carcinomatosis to target the microscopic residual disease after surgical elimination of all macroscopic disease, as was also proposed by other researchers (11).

Papillary thyroid carcinoma is the most common type of histology in the published literature (7). Our patient also had papillary thyroid carcinoma, and a struma ovarii originating from the left ovary, which was larger than $12 \mathrm{~cm}$. Robboy et al. suggested, that $\geq 12 \mathrm{~cm}$ struma ovarii in $88 \%$ were clinically malignant and in patients with papillary carcinoma this number 


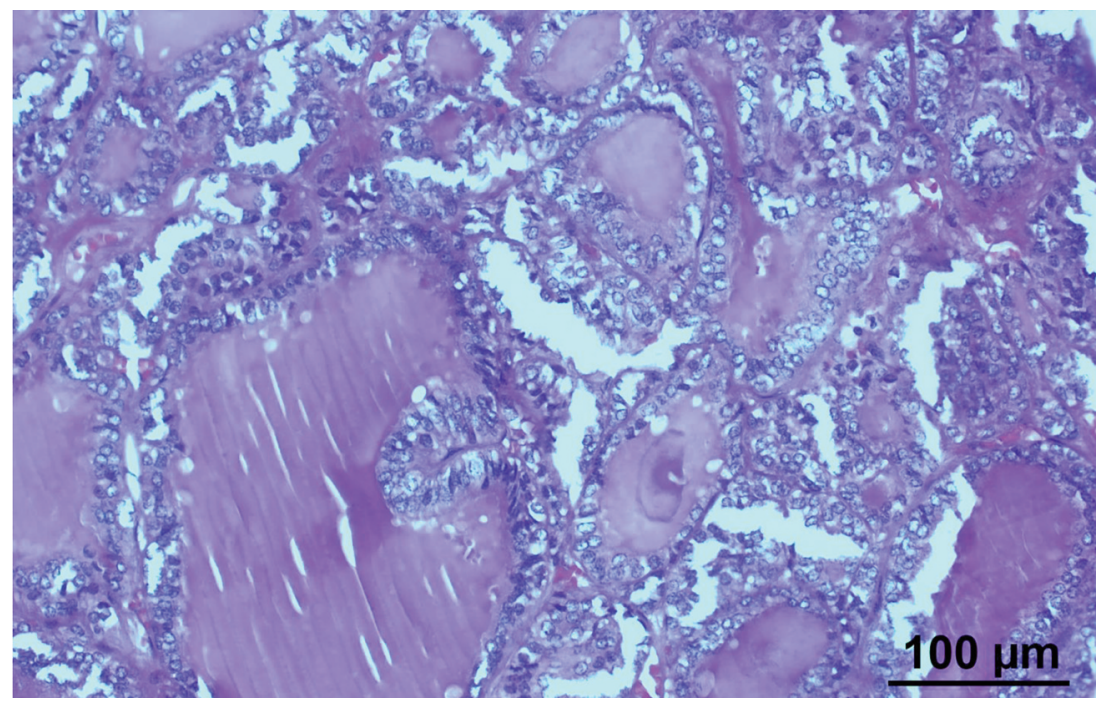

Figure 3. Papillary thyroid cancer embedded in the ovarian struma. Light microscopy 100x magnification.

was $29 \%$ (8). Furthermore, papillary carcinoma histology recurred on average of 4 years earlier than follicular adenomatous neoplasms on average of 11 years (8). In addition, an amount of $33 \%$ of extraovarian dissemination could be found if the strumal component was larger than $12 \mathrm{~cm}$. In line with this, Shaco-Levy et al. found that $\geq 10 \mathrm{~cm}$ tumor size and $\geq 80 \%$ strumal component were associated with rapid progression and increased lethality (12). On the contrary, in the univariate analysis by Li et al. failed to predict either RFS or OS.

According to the FIGO staging principles involvement of the fibrous cortex of the ovary without penetrating involvement of the serosal surface does not upstage the tumor. At our patient, the malignant papillary tumor was embedded in the ovarian struma, and even this latter portion, which was about $15 \mathrm{~cm}$, showed no capsular penetration.

Robboy et al. indicated the fibrous adhesions as adverse features, as well as presence of ascites and large $(\geq 12 \mathrm{~cm})$ strumal tumor size of the ovary, which are suggestive of extraovarian tumor spread at the time of operation and are likely to recur. These features were all present at our patient with evidence of peritoneal metastasis on the preoperative CT record. Despite the fact that most germ cell tumors are chemosensitive, malignant struma ovarii responds poorly to chemotherapy (7); yet our patient responded well to 6 cycles of neoadjuvant chemotherapy, so that no vital extraovarian involvement was detectable on postoperative histology, only distinct fibrosis and scarring, which were originally peritoneal metastasis. As has been suggested by several authors in the literature, these implants may not be malignant peritoneal implants, but extremely well-differentiated "strumosis" corresponding to normal thyroid tissue $(8,13,14)$.

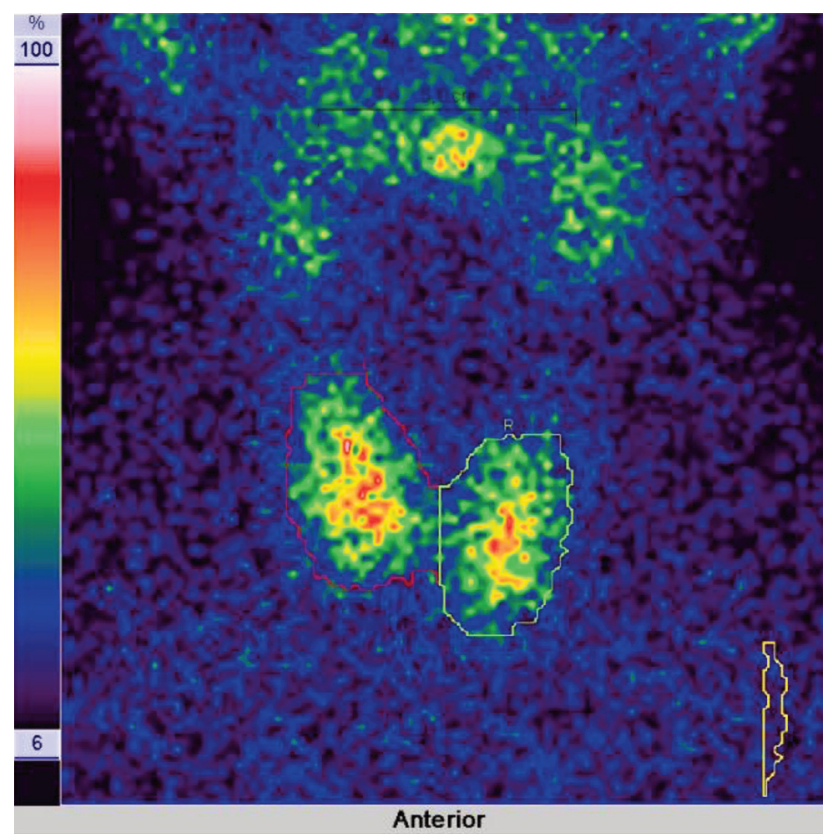

Figure 4. Inhomogeneous, diffuse, reduced accumulation of the thyroid gland without evidence of overactive zones or hypofunctional foci. Thyroid scintigraphy image.

Furthermore, the management of the thyroid gland with thyroidectomy and radioactive iodine ablation after surgical removement of the primary tumor of the ovary is lacking evidence. Some authors recommend routine thyroidectomy followed by an I131 therapy regardless of the presence of 
metastasis at the time of diagnosis to avoid local recurrence and distant metastases $(6,15-17)$. This indication may be questioned since thyroid metastases from extrathyroidal primary tumors are rare and in unselected autopsy series, thyroid metastases were detected in approximately $1 \%$ of the cases $(18,19)$. If extraovarian metastases are present, these usually originate from differentiated thyroid neoplasms, such as papillary carcinoma or follicular carcinoma, and occur in peritoneum, lymph nodes, liver, lung, bone, and brain with manifestation of ascites or pleural effusion (5, 17, 20-22).

According to the German guidelines, surgical removal of thyroid metastases is indicated for extrathyroidal primary tumors if the metastasis is histologically proven or there is diagnostic evidence for it. For this, a local and systemic staging is required $(23,24)$. Our decision was in line with other researchers, who claim that intraabdominal surgical removal of the ovarian tumor and its implants is sufficient, and thyroidectomy and radioiodine therapy are only indicated in the case of metastasis or recurrence (25-27). The indication for thyroidectomy should, therefore, be made within the framework of the interdisciplinary therapy strategy.

In summary, malignant struma ovarii is a very rare histotype of the ovary, which is diagnosed in the vast majority of cases postoperatively and histologically. Due to its rarity and the fact that there is no consensual therapy, multidisciplinary treatment with individualized therapeutic plan involving gynecological oncologists, endocrinologists, surgical oncologists, and nuclear medicine specialists is required. Regular follow-up for at least a decade is recommended since recurrence intervals are quite long (8).

\section{Conflicts of Interest}

The Authors have no conflicts of interest to declare.

\section{Authors' Contributions}

Miklos Acs: conceived the analysis collected the data, wrote the paper. Sebastian Häusler: wrote the paper. Hamid-Reza Lighvani: performed the thyroid scintigraphy, contributed data. Josef Zustin: performed the pathological examination, contributed data. Pompiliu Piso: performed the surgery, wrote the paper.

\section{References}

1 Willemse PH, Oosterhuis JW, Aalders JG, Piers DA, Sleijfer DT, Vermey A and Doorenbos H: Malignant struma ovarii treated by ovariectomy, thyroidectomy, and ${ }^{131} \mathrm{I}$ administration. Cancer 60(2): 178-182, 1987. PMID: 3297279. DOI: 10.1002/10970142(19870715)60:2<178::aid-cncr2820600210>3.0.co;2-q

2 Yannopoulos D, Yannopoulos K and Ossowski R: Malignant struma ovarii. Pathol Annu 11: 403-413, 1976. PMID: 1004942.

3 Fujiwara S, Tsuyoshi H, Nishimura T, Takahashi N and Yoshida Y: Precise preoperative diagnosis of struma ovarii with pseudo-
Meigs' syndrome mimicking ovarian cancer with the combination of ${ }^{131}$ I scintigraphy and ${ }^{18}$ F-FDG PET: case report and review of the literature. J Ovarian Res 11(1): 11, 2018. PMID: 29391043. DOI: 10.1186/s13048-018-0383-2

4 González Aguilera B, Guerrero Vázquez R, Gros Herguido N, Sánchez Gallego F and Navarro González E: The lack of consensus in management of malignant struma ovarii. Gynecol Endocrinol 31(4): 258-259, 2015. PMID: 25539066. DOI: 10.3109/09513590.2014.995616

5 Checrallah A, Medlej R, Saadé C, Khayat G and Halaby G: Malignant struma ovarii: an unusual presentation. Thyroid 11(9): 889-892, 2001. PMID: 11575860. DOI: 10.1089/10507250131 6973163

6 DeSimone CP, Lele SM and Modesitt SC: Malignant struma ovarii: a case report and analysis of cases reported in the literature with focus on survival and I131 therapy. Gynecol Oncol 89(3): 543-548, 2003. PMID: 12798728. DOI: 10.1016/ s0090-8258(03)00141-0

7 Li S, Yang T, Xiang Y, Li X, Zhang L and Deng S: Clinical characteristics and survival outcomes of malignant struma ovarii confined to the ovary. BMC Cancer 21(1): 383, 2021. PMID: 33836675. DOI: $10.1186 / \mathrm{s} 12885-021-08118-7$

8 Robboy SJ, Shaco-Levy R, Peng RY, Snyder MJ, Donahue J, Bentley RC, Bean S, Krigman HR, Roth LM and Young RH: Malignant struma ovarii: an analysis of 88 cases, including 27 with extraovarian spread. Int J Gynecol Pathol 28(5): 405-422, 2009. PMID: 19696610. DOI: 10.1097/PGP.0b013e3181a27777

9 Jean S, Tanyi JL, Montone K, McGrath C, Lage-Alvarez MM and Chu CS: Papillary thyroid cancer arising in struma ovarii. J Obstet Gynaecol 32(3): 222-226, 2012. PMID: 22369392. DOI: $10.3109 / 01443615.2011 .645921$

10 van Driel WJ, Koole SN, Sikorska K, Schagen van Leeuwen JH, Schreuder HWR, Hermans RHM, de Hingh IHJT, van der Velden J, Arts HJ, Massuger LFAG, Aalbers AGJ, Verwaal VJ, Kieffer JM, Van de Vijver KK, van Tinteren H, Aaronson NK and Sonke GS: Hyperthermic intraperitoneal chemotherapy in ovarian cancer. N Engl J Med 378(3): 230-240, 2018. PMID: 29342393. DOI: $10.1056 /$ NEJMoa 1708618

11 Valle SJ, Alzahrani NA, Liauw W, Sugarbaker PH, Bhatt A and Morris DL: Hyperthermic intraperitoneal chemotherapy (HIPEC) methodology, drugs and bidirectional chemotherapy. Indian J Surg Oncol 7(2): 152-159, 2016. PMID: 27065705. DOI: 10.1007/s13193-016-0498-0

12 Shaco-Levy R, Bean SM, Bentley RC and Robboy SJ: Natural history of biologically malignant struma ovarii: analysis of 27 cases with extraovarian spread. Int J Gynecol Pathol 29(3): 212-227, 2010. PMID: 20407319. DOI: 10.1097/PGP.0b013e3181bfb133

13 Brogsitter C, Wonsak A, Würl K and Kotzerke J: Peritoneal strumosis. Eur J Nucl Med Mol Imaging 31(7): 1057, 2004. PMID: 15221212. DOI: 10.1007/s00259-004-1548-3

14 Roth LM and Karseladze AI: Highly differentiated follicular carcinoma arising from struma ovarii: a report of 3 cases, a review of the literature, and a reassessment of so-called peritoneal strumosis. Int J Gynecol Pathol 27(2): 213-222, 2008. PMID: 18317221. DOI: 10.1097/PGP.0b013e318158e958

15 Zhu Y, Wang C, Zhang GN, Shi Y, Xu SQ, Jia SJ and He R: Papillary thyroid cancer located in malignant struma ovarii with omentum metastasis: a case report and review of the literature. World J Surg Oncol 14(1): 17, 2016. PMID: 26791568. DOI: 10.1186/s12957-016-0776-x 
16 Matysiak-Grzes M, Fischbach J, Gut P, Klimowicz A, Gryczynska M, Wasko R and Ruchala M: Struma ovarii maligna. Neuro Endocrinol Lett 34(2): 97-101, 2013. PMID: 23645304.

17 Wu M, Hu F, Huang X, Tan Z, Lei C and Duan D: Extensive peritoneal implant metastases of malignant struma ovarii treated by thyroidectomy and 131I therapy: A case report. Medicine (Baltimore) 97(51): e13867, 2018. PMID: 30572559. DOI: 10.1097/MD.0000000000013867

18 Mortensen J, Woolner LB and Bennett WA: Secondary malignant tumors of the thyroid gland. Cancer 9(2): 306-309, 1956. PMID: 13304848. DOI: 10.1002/1097-0142(195603/04)9:2<306::aidcncr2820090217>3.0.co;2-i

19 Berge T and Lundberg S: Cancer in Malmö 1958-1969. An autopsy study. Acta Pathol Microbiol Scand Suppl (260): 1-235, 1977. PMID: 269649.

20 Meringolo D, Bianchi D, Capula C and Costante G: Papillary thyroid microcarcinoma in struma ovarii. Endocrine 41(1): 164165, 2012. PMID: 22083503. DOI: 10.1007/s12020-011-9564-0

21 Michels A and Haugen B: Malignant struma ovarii. J Clin Endocrinol Metab 95(4): 1505, 2010. PMID: 20375218. DOI: 10.1210/jc.2009-2376

22 Konez O, Hanelin LG, Jenison EL, Goyal M and Randolph W: Functioning liver metastases on an I-131 whole-body scan: a case of malignant struma ovarii. Clin Nucl Med 25(6): 465-496, 2000. PMID: 10836696. DOI: 10.1097/00003072-20000600000014
23 Kim TY, Kim WB, Gong G, Hong SJ and Shong YK: Metastasis to the thyroid diagnosed by fine-needle aspiration biopsy. Clin Endocrinol (Oxf) 62(2): 236-241, 2005. PMID: 15670202. DOI: $10.1111 /$ j.1365-2265.2005.02206.x

24 Deutsche Gesellschaft für Allgemein- und Viszeralchirurgie (DGAV) A. S2k Leitlinie Operative Therapie maligner Schilddrüsenerkrankungen AWMF-Register Nr:088/022, 2012.

25 Zhang $X$ and Axiotis C: Thyroid-type carcinoma of struma ovarii. Arch Pathol Lab Med 134(5): 786-791, 2010. PMID: 20441513. DOI: $10.5858 / 134.5 .786$

26 McGill JF, Sturgeon C and Angelos P: Metastatic struma ovarii treated with total thyroidectomy and radioiodine ablation. Endocr Pract 15(2): 167-173, 2009. PMID: 19289330. DOI: 10.4158/EP.15.2.167

27 Marti JL, Clark VE, Harper H, Chhieng DC, Sosa JA and Roman SA: Optimal surgical management of well-differentiated thyroid cancer arising in struma ovarii: a series of 4 patients and a review of 53 reported cases. Thyroid 22(4): 400-406, 2012. PMID: 22181336. DOI: 10.1089/thy.2011.0162

Received July 14, 2021

Revised August 20, 2021

Accepted September 2, 2021 